\title{
Fabrication and Performances of MTMS/TMCS Bilayer Transparent Superhydrophobic Silica Coating
}

\author{
Jin Wang ${ }^{1, \text { a }}$, Jianjun Chen ${ }^{2, \text { b }}$, Zhipeng Jin ${ }^{1, ~ c}$, Xiaoli \\ $\mathrm{Gu}^{1, \mathrm{~d}}$, Ruizhi Zhang ${ }^{1, \mathrm{e}}$, Jing Huang ${ }^{1, \mathrm{f}}$, \\ Guoqiang Luo ${ }^{1, \mathrm{~g}_{*}}$ \\ ${ }^{1}$ State Key Laboratory of Advanced Technology for
}

\begin{abstract}
The superhydrophobic silica coating has a great performance of self-cleaning which can be applied in the field of energy saving, anti-icing, drag reduction, etc. In this paper, Trimethyltrimethoxysilane (MTMS) /Trimethylchlorosilan (TMCS) superhydrophobic silica coating with two-layer structure was dip-coated. Atomic force microscope (AFM) images show that the surface morphology of MTMS/TMCS silica coating was in micro and nano level, and Sa value was $231 \mathrm{~nm}$, which could achieve good superhydrophobic performance. MTMS/TMCS silica coating has better superhydrophobic performance than MTMS silica coating. The largest contact angle (CA) of MTMS/TMCS silica coating could reach $155.2^{\circ}$, the sliding angle was less than $10^{\circ}$. The MTMS/TMCS silica coating also has good performance of transmittance in the range of visible light and self-cleaning, the transmittance rate could reach more than $95 \%$. All good performances of the MTMS/TMCS silica coating are from its micro and nano surface structure. Modification of TMCS solution would generate a more stable functional group.
\end{abstract}

Keywords-superhydrophobic, self-cleaning, dip-coated, trimethylchlorosilane, micro and nano structure

\section{INTRODUCTION}

There are a lot of superhydrophobic phenomena in the nature, a drop of water on the surface of lotus leaf will roll automatically, which is called lotus leaf effect [1]. Interest in superhydrophobic surfaces (typically defined as having a water contact angle (CA) greater than $150^{\circ}$ and sliding angle less than $10^{\circ}$ ) has grown rapidly in recent years due to unique characteristics such as self-cleaning, antifouling, and fluid drag reduction [2-5].

In order to improve the performance of superhydrophobic coatings, the current research is focused on improving the preparation methods and surface morphology and composition. Various techniques are developed by researchers to fabricate superhydrophobic surfaces, such as self-assembly method, sol-gel method, chemical vapor deposition, electrostatic spinning, dip-coating method and so forth [6-9]. _Method of preparing superhydrophobic coating by dip-coating has been applied multiple times, and some of them have achieved very good results [10-14]. At the same time dipcoating is easy to be operated and don't need expensive equipment. In addition, the formation of micro and nano surface structure and low surface energy materials also improve performance of the coating. The surface chemical modification of silica films using Trimethylchlorosilan
Materials Synthesis and Processing, Wuhan University of Technology, Wuhan 430070, PR China

${ }^{2}$ China Certification \& Inspection Group Hunan Co., LTD, Hunan 410007, PR China wangjin950106@163.com

(TMCS) as a silylating agent has been reported [15-19]. Till date there are only a few reports available on the preparation of water repellent surfaces using Trimethyltrimethoxysilane (MTMS) as a precursor [1923].

In this paper, MTMS/TMCS superhydrophobic silica coatings were fabricated by firstly dip-coated MTMS solution and secondly dip-coated by TMCS solution on the clean glass slide. The surface structure and some performances such as superhydrophobic, transmittance, and self-cleaning were measured.

\section{EXPERIMENTAL AND CHARACTERIZATION}

\section{A. Raw Materials}

Trimethyltrimethoxysilane (MTMS, 98\%) and Trimethylchlorosilane (TMCS) were obtained from Aladdin Industrial Corporation. Methanol (MeOH, AR), Acetone (AR), Hexane, oxalic acid dehydrate (AR) and ammonium hydroxide (AR 25\%) were purchased from Sinopharm Chemical Reagent Co.,Ltd. Dip coating machine was purchased from Qingdao Zhongrui Smart instrument Co.,Ltd.

\section{B. Experimental Processing}

\section{1) Processing glass slide}

The glass slide of $35 \mathrm{~mm} \times 40 \mathrm{~mm}$ was put into the analytically pure ethanol solution for ultrasonic cleaning, which would last for 30 mins to clean the surface of the glass slide.

\section{2) Preparation of MTMS solution}

The molar ratio of $\mathrm{MeOH}$ and MTMS was 35:1, and $1 \mathrm{ml} 0.001 \mathrm{M}$ oxalic acid would be added for the first step catalyzed hydrolysis. The reaction mixture was stirred for $30 \mathrm{~min}$ and then left for $24 \mathrm{~h}$ at room temperature. Following $1.83 \mathrm{ml}$ ammonium hydroxide was added and stirred for 15 min for the second catalyzed hydrolysis, and left for $48 \mathrm{~h}$ for full chemical reaction, which would form white gel. $20 \mathrm{ml}$ methanol was added to the white gel and the mixed solution was sonicated for 1 hour of dispersion.

3) Preparation of TMCS solution

The volume ratio of TMCS and Hexane was 1:19, which was stirred for $30 \mathrm{~min}$.

4) Preparation of MTMS silica coating and MTMS/TMCS silica bilayer coating

The clean glass slide would be dip-coated MTMS solution at $100 \mathrm{~mm} / \mathrm{min}$ to get MTMS silica coating. The glass slide would be immersed in the MTMS solution for 30s, followed by dip-coating TMCS solution at the same 
speed and soaking time to get MTMS/TMCS silica coating. Structure schematic diagram of coatings shown below in Figure 1

a

MTMS silica coating

b

MTMS/TMCS silica coating

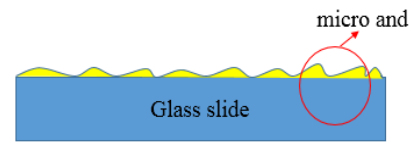

MTMS silica coating

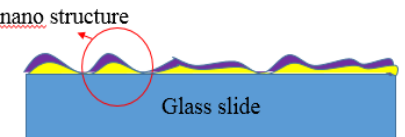

MTMS silica coating

TMCS coating
Figure 1. Structure schematic diagram of (a) MTMS silica coating and (b) MTMS/TMCS silica bilayer coating

\section{Characterization}

The water drop contact angle(CA) was measured using contact angle meter (KrüssEasydrop DSA 20, Germany). Water droplets of $5 \mu \mathrm{L}$ were dropped carefully onto the coating surfaces. The transmittance was evaluated by UV-VIS spectrophotometer (Specord plus, Germany). The roughness and morphology were characterized by atomic force microscopy (AFM) on NANOVER ST400 3-D profiler. The self-cleaning performance was tested by the homemade instrument(the coating surface with a layer of chromium oxide powder)

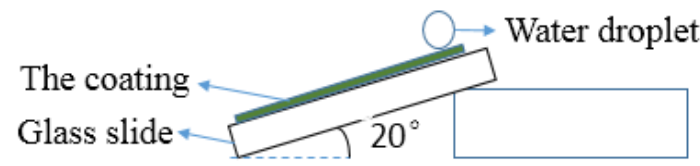

Figure2. Schematic diagram of self-cleaning performance

III. Results and Discussion

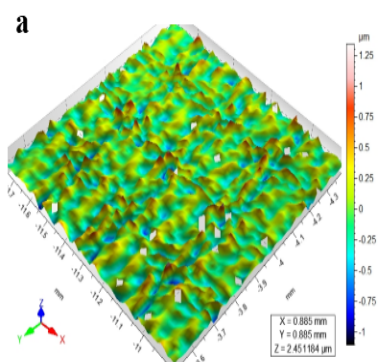

$\mathrm{Sa}=\mathbf{2 3 1 \mathrm { nm }}$ b

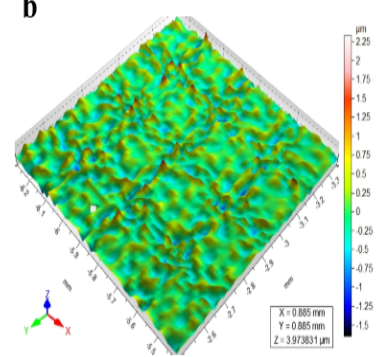

$\mathrm{Sa}=288 \mathrm{~nm}$
Figure 3. The surface roughness and AFM images of the coatings. (a:MTMS/TMCS silica coating; b:MTMS silica coating)..

The AFM results indicated the surface of the coatings were formed similar micro and nano structures, which meet the requirement of superhydrophobic coating. The Sa value of MTMS/TMCS silica bilayer coating was 231nm(Fig. 3(a)), and the Sa value of MTMS silica coating was 288nm (Fig. 3(b)). The surface of
MTMS/TMCS and MTMS coating were both micro and nano level, the surface roughness of them were similar(the Sa represents the roughness of surface).

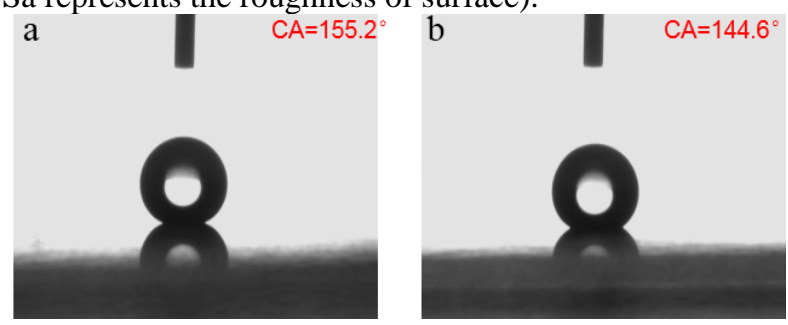

Figure 4. The contact angles of (a) MTMS/TMCS silica coating and (b) MTMS silica coating.

Research shows that low surface energy material are necessary for superhydrophobic coatings and Cassie model shows the theoretical foundation superhydrophobic[24-25]. The MTMS silica coating and MTMS/TMCS silica coating both could achieve superhydrophobic(Fig.4). The contact angle of MTMS/TMCS superhydrophobic silica coating could reach $155.2^{\circ}$ (Fig. 4(a)), while the contact angle of MTMS superhydrophobic silica coating could reached $144.6^{\circ}$ (Fig. 4(b)). MTMS/TMCS silica coating showed better superhydrophobic performance than the MTMS silica coating. Their surface roughness were similar according to the Fig.3. The MTMS/TMCS silica coating showed better superhydrophobic performance of the superhydrophobic coating because the chemical reaction occurred between the TMCS and MTMS could make the numbers of hydrophilic group - $\mathrm{OH}$ down, which could to generate a more stable $\mathrm{Si}-\mathrm{O}$ bond to make the contact angle larger[19].

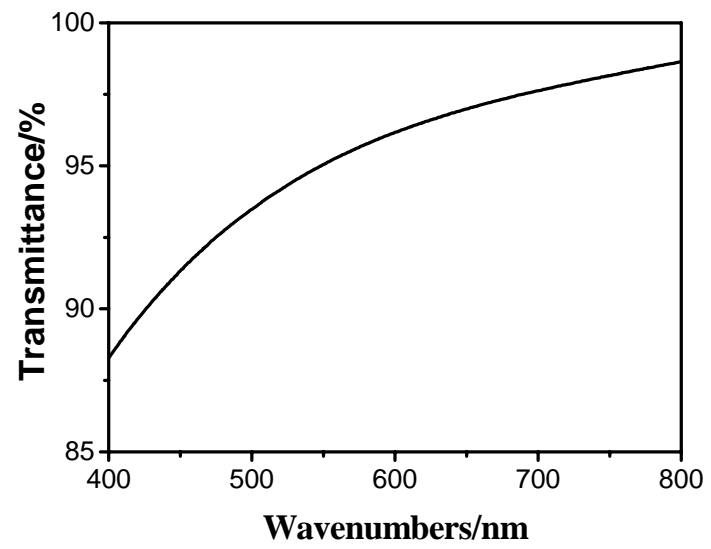

Figure 5. Transmittance of MTMS/TMCS coating in the range of $400 \mathrm{~nm}-800 \mathrm{~nm}$. 


\section{MTMS/TMCS silica coating}

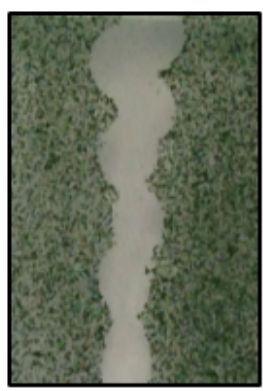

a

Figure 6. Self-cleaning effect diagram of MTMS/TMCS silica coating (a:MTMS/TMCS silica coating, b:clean slide).

The MTMS/TMCS silica coating showed the good transmittance in the visible range from $400 \mathrm{~nm}$ to $800 \mathrm{~nm}$, the largest transmittance was beyond 95\% (Fig.5). Two drops of water were dropped on both slides covered with Chromium oxide powder. A great difference could be seen between the MTMS/TMCS silica coating and clean slide (Fig.6). The slide with MTMS/TMCS silica coating could clean the powder automatically, which indicated the good self-cleaning of the MTMS/TMCS silica coating.

\section{CONCLUSION}

In this study, a MTMS/TMCS superhydrophobic silica bilayer coating with good transmission of light and selfcleaning, was formed by dip-coating method. The MTMS/TMCS silica coating had micro and nano structure which lead to good superhydrophobic. MTMS/TMCS silica bilayer coating showed better superhydrophobic than the MTMS silica coating, the largest contact angle of MTMS/TMCS coating could reach $155.2^{\circ}$. And the MTMS/TMCS silica coating showed good transmittance rate, which could reach more than 95\%, and a good performance of self-cleaning.

\section{ACKNOWLEDGEMENTS}

This work has been supported by National Natural Science Foundation of China (51202175), the 111 Project (B13035), National Natural Science Foundation of Hubei Province(2014CFB257 and 2014CFB258) and the Fundamental Research Funds for the Central Universities (WUT: 2013-II-024 and 2014-II-15).

\section{REFERENCES}

[1] Jiang L, Zhao Y, Zhai J. A Lotus-Leaf-like Superhydrophobic Surface: A Porous Microsphere/Nanofiber Composite Film Prepared by Electrohydrodynamics[J]. Angewandte Chemie, 2004, 116(33): 4438-4441.

[2] Nosonovsky M, Bhushan B. Multiscale dissipative mechanisms and hierarchical surfaces: friction, superhydrophobicity, and biomimetics [M]. Springer, 2008.

[3] Bhushan B. Nanotribology and nanomechanics [M]. Berlin: Springer, 2011.

[4] Bhushan B. Bioinspired structured surfaces [J]. Langmuir, 2012, 28 (3): 1698-1714.
[5] Ebert D, Bhushan B. Transparent, superhydrophobic, and wearresistant coatings on glass and polymer substrates using $\mathrm{SiO} 2, \mathrm{ZnO}$, and ITO nanoparticles [J]. Langmuir, 2012, 28 (31): 11391-11399.

[6] Sun T, Tan H, Han D, et al. No platelet can adhere - largely improved blood compatibility on nanostructured superhydrophobic surfaces [J]. Small, 2005, 1(10): 959-963.

[7] Jiang Y, Wan P, Smet M, et al. Self-Assembled Monolayers of a Malachite Green Derivative: Surfaces with $\mathrm{pH}$-and UV-Responsive Wetting Properties [J]. Advanced materials, 2008, 20(10): 19721977.

[8] Celestini F, Kofman R, Noblin X, et al. Water jet rebounds on hydrophobic surfaces: a first step to jet micro-fluidics [J]. Soft Matter, 2010, 6(23): 5872-5876.

[9] Guo Z, Liu W, Su B L. Superhydrophobic surfaces: from natural to biomimetic to functional [J]. Journal of colloid and interface science, 2011, 353(2): 335-355.

[10] Roach P, Shirtcliffe N J, Newton M I. Progess in superhydrophobic surface development [J]. Soft Matter, 2008, 4(2): 224-240.

[11] Nguyen D D, Tai N H, Lee S B, et al. Superhydrophobic and superoleophilic properties of graphene-based sponges fabricated using a facile dip coating method[J]. Energy \& Environmental Science, 2012, 5(7): 7908-7912.

[12] Farhadi S, Farzaneh M, Kulinich S A. Anti-icing performance of superhydrophobic surfaces [J]. Applied Surface Science, 2011, 257(14): 6264-6269.

[13] Zhang G, Wang D, Gu Z Z, et al. Fabrication of superhydrophobic surfaces from binary colloidal assembly [J]. Langmuir, 2005, 21(20): 9143-9148.

[14] Zhai L, Cebeci F C, Cohen R E, et al. Stable superhydrophobic coatings from polyelectrolyte multilayers [J]. Nano Letters, 2004, 4(7): 1349-1353.

[15] Wang J, Zhang C R, Feng J. Modification of nanoporous silica film by trimethylchlorosilane[J]. Acta Phys. Chim. Sin, 2004, 20: 1399.

[16] Grobelny J P, Celichowski G, Cichomski M, et al. Comparison of two methods of methyl group grafting to the silica thin film surface and its tribological properties measured by atomic force microscopy [J]. Tribology Letters, 2004, 16(3): 181-185.

[17] He Z W, Zhen C M, Liu X Q, et al. Microstructural characterization of low dielectric silica xerogel film [J]. Thin Solid Films, 2004, 462: 168-171.

[18] Huang K, He Z, Chao K. Mesoporous silica films-characterization and reduction of their water uptake [J]. Thin solid films, 2006, 495(1): 197-204.

[19] Venkateswara Rao A, Latthe S S, Nadargi D Y, et al. Preparation of MTMS based transparent superhydrophobic silica films by sol-gel method [J]. Journal of colloid and interface science, 2009, 332(2): 484-490.

[20] Khummalai N, Boonamnuayvitaya V. Suppression of arsenopyrite surface oxidation by sol-gel coatings [J]. Journal of bioscience and bioengineering, 2005, 99(3): 277-284.

[21] Liu Y, Chen X, Xin J H. Super-hydrophobic surfaces from a simple coating method: a bionic nanoengineering approach [J]. Nanotechnology, 2006, 17(13): 3259.

[22] Smitha S, Shajesh P, Mukundan P, et al. Synthesis of biocompatible hydrophobic silica-gelatin nano-hybrid by sol-gel process [J]. Colloids and surfaces B: Biointerfaces, 2007, 55(1): 38-43.

[23] Kim B R, Kang J W, Lee K Y, et al. Physical properties of low-k films based on the co-condensation of methyltrimethoxysilane with a bridged silsesquioxane [J]. Journal of materials science, 2007, 42(12): 4591-4602.

[24] Cassie, A. B. D. Contact angle [J]. Discuss Faraday Soc. 1948, 3: 11-15

[25] Cassie A B D, Baxter S. Wettability of porous surfaces [J]. Transactions of the Faraday Society, 1944, 40: 546-551. 